\title{
Research on risk assessment of high-speed railway operation based on network ANP
}

\author{
Leyi Cheng, Yinghan Wang and Yichuan Peng \\ The Key Laboratory of Road and Traffic Engineering, Ministry of Education, \\ Tongji University, Shanghai, China
}

High-speed railway operation

\begin{abstract}
Purpose - The causes of high-speed railway failures are complex, and it is difficult to quantitatively and accurately describe safety evaluation. The purpose of this paper is to construct a model to ensure the safety of high-speed railway operations.

Design/methodology/approach - The authors construct a high-speed railway operation safety evaluation index system from four aspects: personnel, equipment, environment and management and analyze the inter-coupling relationship of various safety factors. Based on the evaluation index system, the use of network analytic hierarchy process (ANP) and fuzzy comprehensive evaluation will be used to establish a high-speed railway operation safety evaluation model.

Findings - Through the literature investigation and field investigation, combined with high-speed railway safety key points and system composition, 4 first-level indicators and 17 second-level indicators were selected to construct a high-speed railway operation safety evaluation index system. It can be seen from the results that the personnel management system and the signal and control system have the largest weight.

Originality/value - On the basis of establishing an evaluation index system, comprehensively considering the internal coupling relationship between evaluation indexes and the fuzziness of high-speed railway operation safety evaluation, high-speed railway uses ANP fuzzy network analysis method to construct highspeed railway operation, and the safety evaluation model has certain advantages and practicability in the case of the relative lack of high-speed railway operation data and fault data.
\end{abstract}

Keywords High-speed railway, Influencing factors, Fuzzy comprehensive evaluation,

Network analytic hierarchy process, Safety evaluation

Paper type Research paper

\section{Introduction}

As of the end of 2018, the mileage of China's high-speed railways that have been built and put into operation reached more than $29,000 \mathrm{~km}$, accounting for more than $66 \%$ of the world's high-speed railway operating mileage. Although China's high-speed railway construction has made world-renowned achievements, it still needs to be improved in terms

(C) Leyi Cheng, Yinghan Wang and Yichuan Pe. Published in Smart and Resilient Transportation. Published by Emerald Publishing Limited. This article is published under the Creative Commons Attribution (CC BY 4.0) licence. Anyone may reproduce, distribute, translate and create derivative works of this article (for both commercial and non-commercial purposes), subject to full attribution to the original publication and authors. The full terms of this licence maybe seen at http:// creativecommons.org/licences/by/4.0/legalcode

This research has been supported by projects of National Key R\&B Program of China (No. 2018YFB201403). 
SRT

3,1

38

of operation management and safety assurance. High-speed railway operation safety assessment and risk management have received more and more value and attention.

As an important part of system engineering, safety evaluation aims to make a quantitative estimate or qualitative description of the damage or potential risk sources that the system or engineering may suffer in operation. The high-speed railway system is complicated in composition, and the normal operation of its power supply system, signal and communication system, wheel-rail system and control system are all related to the safe operation of the train. In addition, high-speed railways are also vulnerable to weather conditions, human interference and other factors during operation. At present, many experts and scholars in the field of safety at home and abroad use different methods and models to study and analyze the safety of high-speed railway operations: Guo et al. (2016) studied the impact of the personality characteristics of high-speed rail train drivers on driving safety, using the NEO personality scale and 221 high-speed train drivers of the original Beijing Railway Bureau were surveyed by questionnaires in this way, and the results of the survey were analyzed by modeling. Qian et al. (2019) used FLAC3D modeling software to analyze the stratum settlement characteristics when the high-speed railway line and the subway tunnel intersect at different angles, to study the impact of stratum settlement on the safety of the highspeed railway during tunnel excavation; Xu et al. (2019) analyzed the impact of axle fatigue damage on high-speed railway operation safety. The impact of railway operation safety is evaluated by analyzing the depth of scratches on the axle surface to evaluate the impact of scratches on fatigue performance. Shi and Jian (2018) used the analytic network process (ANP) and evidence theory to evaluate the risk level of wireless block centers. The above research mainly focuses on the impact of personnel, external environment, key equipment and other single factors on the safety of high-speed railways, without systematically summarizing and analyzing the risk factors. Lisha et al. (2015) established a safety situation evaluation model based on the construction of an urban rail transit station safety situation evaluation index system, using analytic hierarchy process (AHP) and entropy method. However, the AHP method ignores the coupling relationship between the evaluation indicators and is not completely applicable to the complex high-speed railway system. As an improvement and upgrade method of the AHP method, the ANP takes the interaction and mutual influence between indicators into account and has a wider applicability. ANP method has been more maturely used in risk evaluation (Wang and Jianbin, 2019), project bidding (Chen et al, 2018), capability evaluation (Wu et al., 2018; Qin, 2018; He et al., 2018) and other fields.

Combined with the research foundation of relevant safety evaluation methods, the ANP is used to first sort out the many safety factors that affect the safe operation of high-speed railways, summarize the safety factor index system and systematically analyze the coupling relationship between the safety factors, and then use the ANP to obtain the contribution degree of each safety factor to the operation safety of the high-speed railway, and finally uses the fuzzy comprehensive evaluation method to make a comprehensive evaluation of the operation safety level of the high-speed railway.

\section{Analysis of safety index of high-speed railway}

Through consulting related literature (Sishuai, 2011; Feng et al., 2013) and going to several railway bureau group companies for field investigations, the factors affecting the safety of high-speed railway operations are divided into four aspects: personnel, equipment, environment and management. The four factors are coupled and influence each other, and jointly affect the safety of high-speed railway operations.

\subsection{Evaluation index analysis}

The factors affecting the safety of high-speed railway operations are analyzed from the four aspects of personnel, equipment, environment and management. 
Personnel factors not only refer to the staff of various functional departments such as train depot, locomotive depot, public works depot and electricity depot during the operation of high-speed railway, but also include management personnel and technical operators at all levels, as well as passengers of high-speed rail trains. The staff is the main body of various tasks. The staff's safety literacy, business ability, vocational skills, response capability and emergency response capability in response to emergencies all affect the safe driving of high-speed railways. Combining the work characteristics of each train station on the high-speed railway, the safety factors of staff are divided into safety literacy and business level. Passengers are the main body of high-speed rail transportation services. Passengers' improper behavior when taking the high-speed rail trains, such as smoking triggering a smoke alarm and causing the train to stop temporarily, will also affect the safe operation of high-speed rail trains.

There are many types of equipment involved in the operation of high-speed railways, with high complexity and coupling. At the same time, the working environment of high-speed railways is outdoor, and various equipment are easily affected by natural environmental factors. Therefore, ensuring the normal operation and good operation of the equipment is the key to ensuring driving safety. The fixed infrastructure and mobile equipment involved in the operation of high-speed railways include tracks, wheel-rail systems, traction power supply systems, signal systems and communication systems. The track is a fixed infrastructure for high-speed railway operation. The smoothness of the track and track surface conditions directly affect the stability of the train and the comfort of passengers. The traction power supply system transports electric energy for electrified railways, and the main equipment includes the catenary system, traction substation and data acquisition and monitoring and control system. Among them, the catenary system is laid in the open air along the railway and has a complex structure, so the failure rate is higher. The high-speed train signal and control system is an important guarantee for the safety and efficiency of trains. It mainly includes the train control system, the dispatching centralized system and the station interlocking. The railway communication system is a system that realizes special railway communication services. The current high-speed railway communication system uses the GSM-R digital mobile communication system that has stricter requirements in terms of wireless field strength coverage, network service quality and reliability.

Environmental factors can be divided into natural environment and social environment. The natural environment includes weather conditions (including strong winds, rainstorms, blizzards, etc.), geological disasters (including mudslides, landslides, earthquakes, etc.), special soil sections (collapsible loess, expansive soil, etc.), etc. The social environment refers to the political, legal, economic and public security environment in social operations.

Management plays the role of planning, leading, organizing and coordinating in the operation of high-speed railways. A scientific and reasonable management system can make various tasks more efficient and orderly, thereby ensuring the safety of train operations. Combined with the characteristics of high-speed railway operations, the management system can be divided into personnel management system and equipment management system.

Based on the above analysis, the four main aspects of safety factors are further divided to construct a high-speed railway operation safety evaluation index system (Figure 1), including 4 first-level safety indicators and 17 second-level safety indicators, for the establishment of a safety evaluation model basis.

\subsection{Analysis of coupling relationship of evaluation indexes}

After the evaluation index system is established, the coupling relationship between the indexes should be analyzed and judged. The evaluation indicators are not independent of each other, but there are certain dependencies and feedback relationships. For example, the staff's low level of business may lead to improper 
3,1

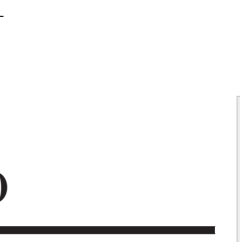

Figure 1.

High-speed railway operation safety evaluation indicators

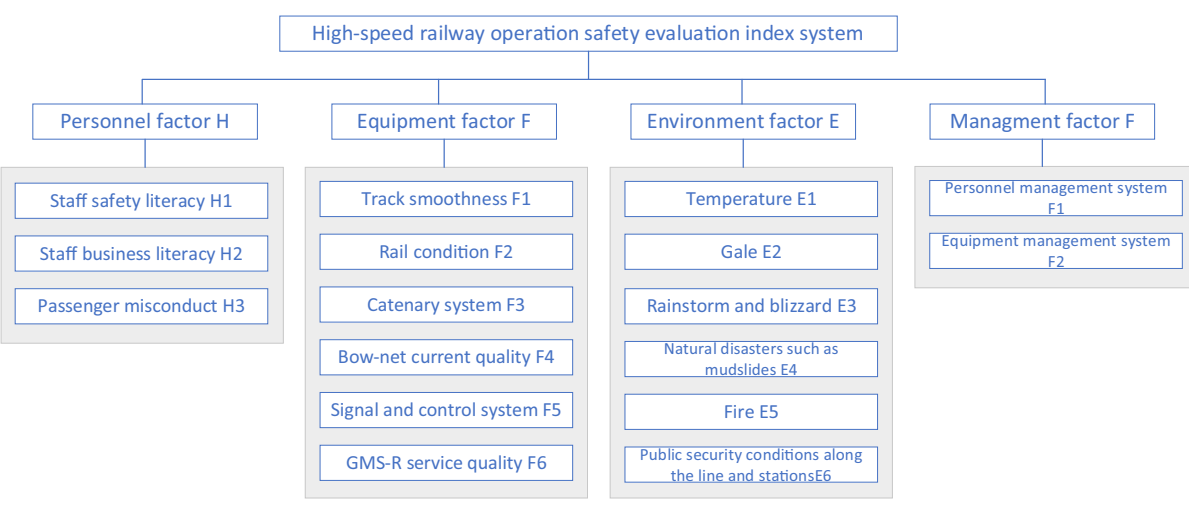

operation of the equipment and cause vehicle failure; when the temperature changes, the tension and sag of the catenary will change with the thermal expansion and contraction, which will affect the quality of bow net of the flow; the public security disorder at the station may cause fire safety hazards; the control of the train system will take emergency measures such as automatic speed reduction and temporary parking to avoid safety accidents when encountering disasters such as heavy rain or snow or a collapse in front. By referring to relevant materials (Renchao and Dengbo, 2017) and consulting experts and scholars in related fields, the coupling relationship between the evaluation indicators is determined in Table 1.

\section{Evaluation model construction}

\subsection{Network analytical process}

The ANP is a comprehensive system evaluation method developed by T.L.Saaty on the basis of the AHP. The structure of ANP mainly consists of two parts, one is the control layer, which contains

\begin{tabular}{lll}
\hline Impact indicators & & Affected indicators \\
\hline Personnel factor $H$ & $H_{1}$ & $H_{2}, E_{5}, E_{6}$ \\
& $H_{2}$ & $H_{1}, F_{1}, F_{2}, F_{3}, F_{4}, F_{5}, F_{6}, E_{5}$ \\
Equipment factor $F$ & $H_{3}$ & $F_{2}, F_{5}, E_{5}, E_{6}$ \\
& $F_{1}$ & $F_{1}, F_{2}, F_{4}$ \\
& $F_{2}$ & $F_{1}, F_{2}, F_{4}$ \\
$F_{3}$ & $F_{4}, F_{6}, E_{5}$ \\
Environment factor $E$ & $F_{4}$ & $F_{3}, F_{6}$ \\
& $F_{5}$ & $F_{5}, F_{6}, E_{1}, E_{2}, E_{3}, E_{4}, E_{5}, E_{6}$ \\
$F_{6}$ & $F_{5}$ \\
$E_{1}$ & $F_{1}, F_{3}, E_{2}, E_{5}$ \\
& $E_{2}$ & $F_{3}, E_{5}$ \\
$E_{3}$ & $F_{2}, F_{3}, F_{6}, E_{4}, E_{5}$ \\
Management factor $M$ & $E_{4}$ & $F_{1}, F_{2}, F_{3}, F_{6}$ \\
& $E_{5}$ & $F_{2}, F_{3}, F_{4}, E_{6}$ \\
$E_{6}$ & $F_{2}, E_{5}, E_{6}$ \\
$M_{1}$ & $H_{1}, H_{2}, E_{6}$ \\
$M_{2}$ & $F_{1}, F_{2}, F_{3}, F_{4}, F_{5}, F_{6}$ \\
\hline
\end{tabular}

Table 1.

Evaluation indicators Management factor $M$ coupling relationship 


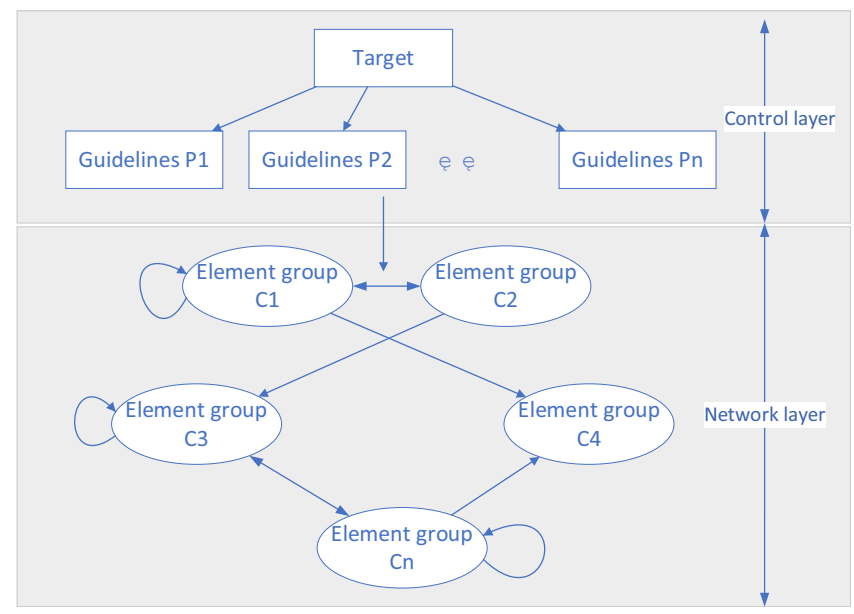

High-speed railway operation

Figure 2.

ANP typical structure

goals and guidelines; the other is the network layer, the elements of the network layer are not independent, there is interaction and mutual influence (Figure 2). Compared with AHP, the hierarchical structure of ANP is more complex, not only a hierarchical structure, and at the same time, it also considers the loop and feedback between hierarchical structures, as well as the interdependence and dominance of internal elements at the same level. Therefore, for high-speed railway systems with complex obstacle causes and a coupling relationship between safety factors, ANP is used to determine the relative importance of each evaluation index, that is, the size of the weight, which is more suitable and advantageous than AHP.

Assuming that there are $N$ element groups in the network layer, first take $e_{i k}(i=1 \ldots N$; $k=1 \ldots n)$ in the element group $C_{i}$ as the sub-criteria to construct the element $e_{j l}(j=1 \ldots n$; $1=1 \ldots m)$ to compare the relative importance of $e_{i k}$, that is, the judgment matrix, and the ranking vector $\left(w_{j 1}^{(i k)}, w_{j 2}^{(i k)}, \ldots, w_{j l}^{(i k)}, \ldots, w_{j m}^{(i k)}\right)^{T}$ is obtained by the characteristic root method, and $\sum w_{j i}^{(i k)}=1$. By analogy, the ranking vector of the elements in the element group $C_{j}$ to all the elements in $C_{i}$ can be obtained, so that we can obtain the ranking matrix $W_{i j}$. If the elements in the element group $C_{i}$ are not affected by $C_{j}$, then $W_{i j}=0$ :

$$
W_{i j}=\left[\begin{array}{cccccc}
w_{j 1}^{(i 1)} & w_{j 1}^{(i 2)} & \cdots & w_{j 1}^{(i k)} & \cdots & w_{j 1}^{(i n)} \\
w_{j 2}^{(i 1)} & w_{j 2}^{(i 2)} & \cdots & w_{j 2}^{(i k)} & \cdots & w_{j 2}^{(i n)} \\
\vdots & \vdots & \vdots & \vdots & \vdots & \vdots \\
w_{j l}^{(i 1)} & w_{j l}^{(i 2)} & \cdots & w_{j l}^{(i k)} & \cdots & w_{j l}^{(i n)} \\
\vdots & \vdots & \vdots & \vdots & \vdots & \vdots \\
w_{j m}^{(i 1)} & w_{j m}^{(i 2)} & \cdots & w_{j m}^{(i k)} & \cdots & w_{j m}^{(i n)}
\end{array}\right]
$$


SRT

3,1

There are $N \times N$ such sorting matrices, and the super-matrix $W$ is obtained by forming subblocks of the sorting matrix. The core work of ANP is to solve the super matrix:

$$
W=\left[\begin{array}{cccc}
W_{11} & W_{12} & \ldots & W_{1 N} \\
W_{21} & W_{22} & \ldots & W_{2 N} \\
\vdots & \vdots & \vdots & \vdots \\
W_{N 1} & W_{N 2} & \cdots & W_{N N}
\end{array}\right]
$$

It can be seen from the above definition that the column vector of the super matrix sub-block $W_{i j}$ is normalized, but the column vector of the super matrix $W$ is not normalized. Therefore, it is also necessary to perform weighting processing on the super matrix, that is, to compare the element groups in pairs to obtain the judgment matrix and pass the consistency test, and obtain the normalized eigenvector by the eigen-root method, thereby obtaining the weighting matrix $A^{\prime}$ :

$$
A^{\prime}=\left[\begin{array}{cccc}
a_{11} & a_{12} & \ldots & a_{1 N} \\
a_{21} & a_{22} & \ldots & a_{2 N} \\
\vdots & \vdots & \vdots & \vdots \\
a_{N 1} & a_{N 2} & \cdots & a_{N N}
\end{array}\right]
$$

From $-W=A \times W$, the weighted hypermatrix $-W$ is obtained. Finally, when $W^{\infty}=\lim _{t \rightarrow \infty}-W^{t}$ exists, the limit supermatrix $W^{\infty}$ is obtained.

\subsection{Fuzzy comprehensive evaluation}

In the objective world, there are many phenomena with uncertain nature, and these undefined phenomena are called fuzzy phenomena. In engineering applications, there are also a large number of factors and indicators that are restricted by various factors and difficult to quantify. Fuzzy comprehensive evaluation is based on fuzzy mathematics, according to the theory of membership degree and the principle of fuzzy composite operation, quantitatively evaluate many indicators with unclear boundaries, and then make a systematic overall evaluation of fuzzy phenomena (Du et al., 2015).

First of all, it is necessary to establish the factor set $U$ and the comment set $V$ of the evaluation object based on experience and relevant research data. $U=\left\{u_{1}, u_{2}, \ldots, u_{m}\right\}, m$ is the number of evaluation indicators; $V=\left\{v_{1}, v_{2}, \ldots, v_{m}\right\}, n$ is the number of evaluation levels.

According to the membership degrees of $\mathrm{m}$ evaluation factors to $n$ evaluation levels, a fuzzy relationship matrix $R$ is constructed:

$$
R=\left(\left(r_{i j}\right)_{m^{*} n}\right)=\left[\begin{array}{cccc}
r_{11} & r_{12} & \ldots & r_{1 n} \\
r_{21} & r_{22} & \ldots & r_{2 n} \\
\vdots & \vdots & \vdots & \vdots \\
r_{m 1} & r_{m 2} & \cdots & r_{m n}
\end{array}\right]
$$

In the formula, $r_{i j}$ is the degree of membership of the evaluation factor $u_{i}$ on the evaluation level $v_{j}$, that is, the frequency distribution, which is generally normalized so that $\Sigma r_{i j}=1$. 
After obtaining the fuzzy relationship matrix $R$, it is necessary to judge the relative importance of each evaluation factor, and obtain the evaluation factor weight distribution set $A, A=\left\{a_{1}, a_{2}, \ldots, a_{i}, \ldots, a_{m}\right\}$, where $a_{i}$ represents the proportion of evaluation factor $u_{i}$ in the factor set, and $\Sigma a_{i}=1$.

Use the weight vector $A$ to perform fuzzy synthesis operation on the fuzzy relationship matrix to obtain the fuzzy subset $B$ of the membership degree of the evaluation target at each evaluation level, $B=A \times R=\left\{b_{1}, b_{2}, \ldots, b_{j}, \ldots, b_{n}\right\}$, where $b_{j}$ represents the membership degree of the evaluation target on the evaluation level $v_{j} \times$ is the symbol of composite operation. Generally, there are different fuzzy composite operation methods such as search operator and ordinary matrix multiplication.

According to the principle of maximum degree of membership, the final evaluation result is obtained, and the largest in $b_{j}$ is the evaluation level of the evaluation target.

\section{High-speed railway operation risk assessment method based on analytic network process}

The ANP considers the interaction and mutual influence between indicators and then determines the weight of each safety factor, taking into account the complexity of the evaluation object. According to the analysis of the coupling relationship between the evaluation factors, the ANP network layer structure is established (Figure 3).

\subsection{Determination of the weights of first-level risk factors}

According to the Saaty 1-9 scale (Table 2), and compare the relative importance of the firstlevel evaluation factors in pairs, the judgment matrix is constructed according to expert judgment (Table 3). Through the consistency test, the weight of each factor is obtained.

\subsection{Determination of the weights of second-level risk factors}

Using the Saaty1-9 scale table, the relative importance of each evaluation factor is compared in pairs by means of expert scoring, and a pairwise comparison matrix is obtained. Then use the decision-making software Super Decision to establish a judgment matrix and pass the consistency test. The ranking vector is obtained by the eigen-root

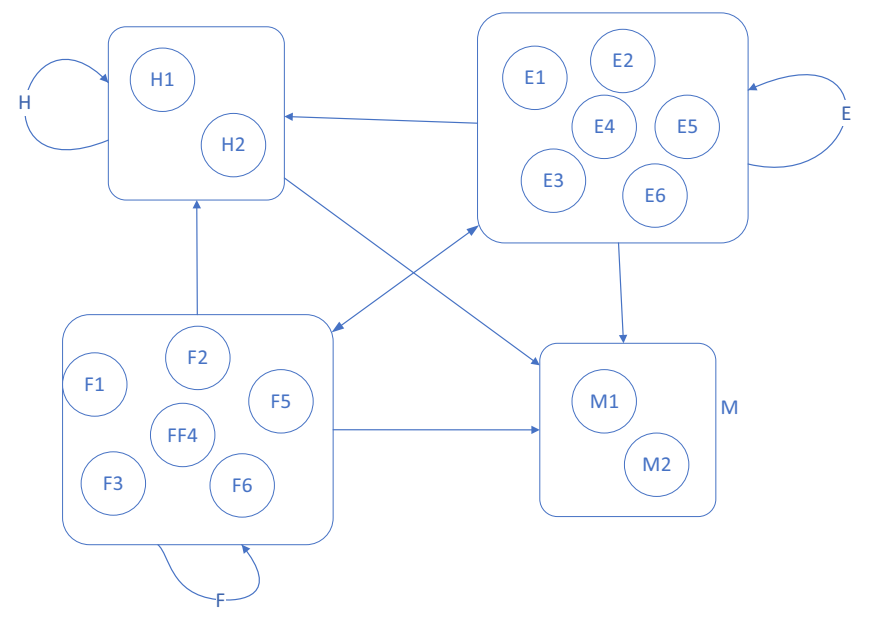

High-speed railway operation 
SRT

3,1

method, and then establish the ranking matrix, then the unweighted super matrix is calculated (Table 4).

The weighted super matrix is obtained by weighting the corresponding secondary evaluation factors (Table 5). Finally, perform the limit operation on the weighted super matrix to obtain the limit super matrix (Table 6). The weights of the primary evaluation factors and secondary evaluation factors are shown in Figures 4 and 5, respectively. After normalizing the weight of each evaluation factor, the weight vector $A$ of the factor set is obtained:

$$
\begin{aligned}
A= & (0.051,0.108,0.053,0.011,0.006,0.044,0.046,0.180,0.114,0.013,0.007 \\
& 0.046,0.008,0.021,0.009,0.235,0.049)
\end{aligned}
$$

It can be seen from Figure 4 that the first-level factor that has the greatest impact on the safety of high-speed railway operations is the equipment factor, followed by the management factor. The high-speed railway system has a complex structure and high degree of coupling and involves many types of equipment elements and a high degree of automation. Therefore, ensuring the normal operation of equipment is the key to ensuring the safety of high-speed railway operations; management elements are the dominant factors for overall coordination of personnel, equipment, environment and other factors, and management is the dominant factor in the overall planning of man-machine-environment. Therefore, a sound, scientific, reasonable and efficient management system can reduce the risk of the other three safety factors, thereby ensuring the safety of high-speed rail.

From Figure 5, we can see that the two secondary factors, the personnel management system and the signal and control system, have the greatest impact on the safety of highspeed railway operations. The personnel management system ensures that the staff of

\begin{tabular}{|c|c|c|c|}
\hline & Serial number & Importance level & Value \\
\hline & 1 & $i$ and $j$ are equally important & 1 \\
\hline & 2 & The $i$ element is slightly more important than the jelement & 3 \\
\hline & 3 & The $i$ element is obviously more important than the $j$ element & 5 \\
\hline & 4 & The $i$ element is more important than the $j$ element & 7 \\
\hline & 5 & The $i$ element is extremely important than the $j$ element & 9 \\
\hline & 6 & The $i$ element is slightly less important than the $j$ element & $1 / 3$ \\
\hline & 7 & The $i$ element is obviously less important than the $j$ element & $1 / 5$ \\
\hline Table 2. & 8 & The $i$ element is less important than the $j$ element & $1 / 7$ \\
\hline Saaty 1-9 scale table & 9 & The $i$ element is extremely less important than the $j$ element & $1 / 9$ \\
\hline
\end{tabular}
various departments and levels carry out various tasks in a safe, orderly and efficient

Table 3.

First-level risk factor weight judgment matrix

\begin{tabular}{lllcl}
\hline Risk factor & \multicolumn{1}{c}{$H$} & $F$ & $E$ & $M$ \\
\hline$H$ & 0.250 & 0.208 & 0.084 & 0 \\
$F$ & 0 & 0.462 & 0.438 & 0 \\
$E$ & 0 & 0.251 & 0.423 & 0 \\
$M$ & 0.750 & 0.078 & 0.055 & 0 \\
\hline
\end{tabular}




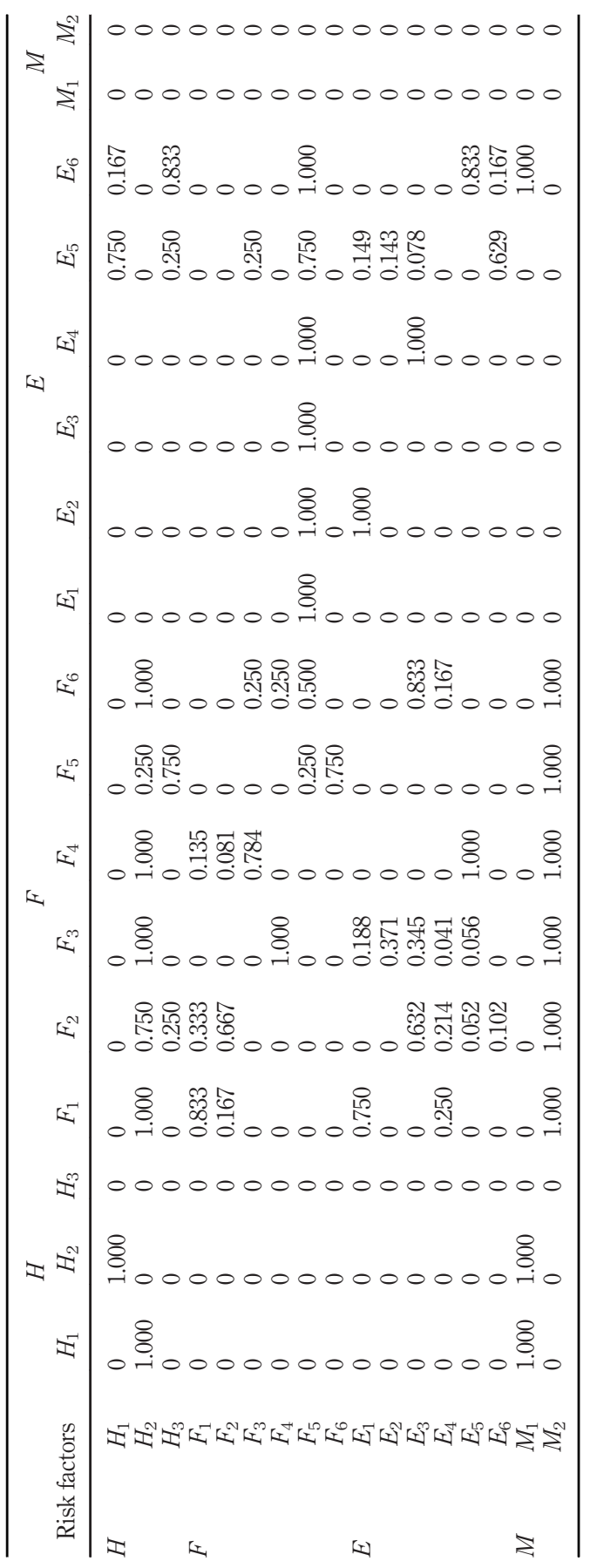

High-speed railway operation

Table 4. Unweighted overrun super matrix of secondary risk factors relative to primary risk factors 
SRT

3,1

46

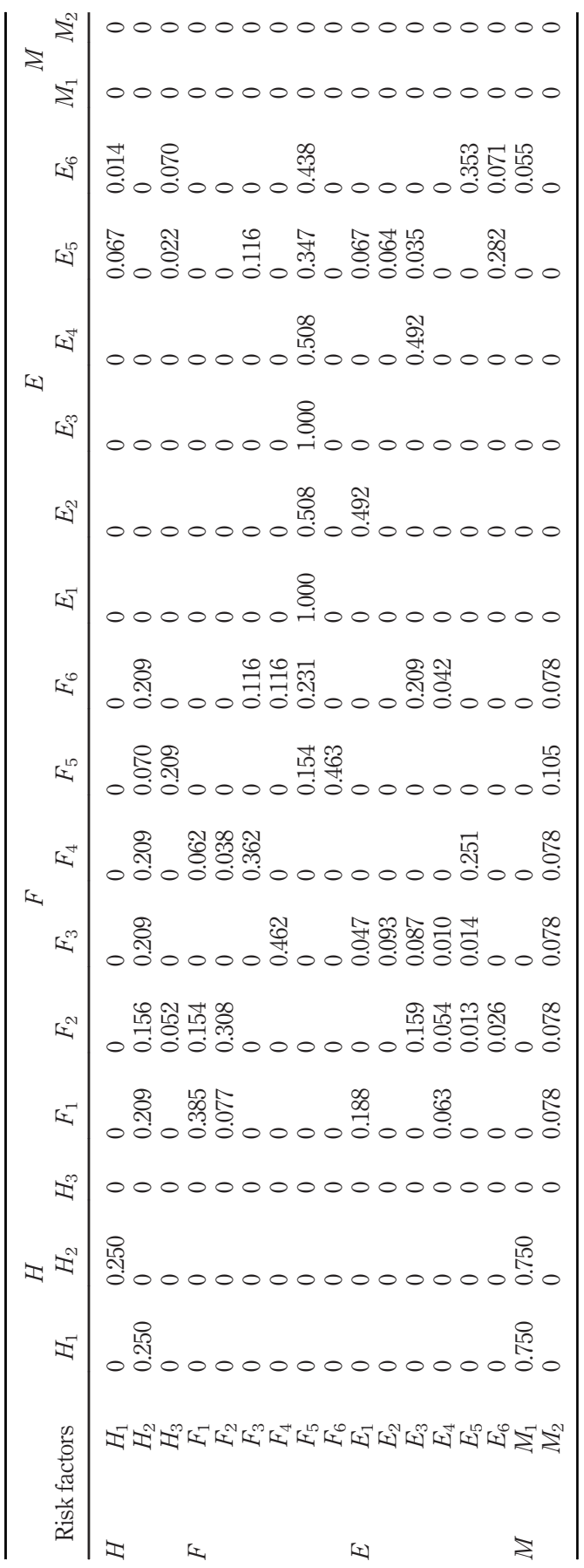

Table 5.

Weighted overrun super matrix of secondary risk factors relative to primary risk factors 


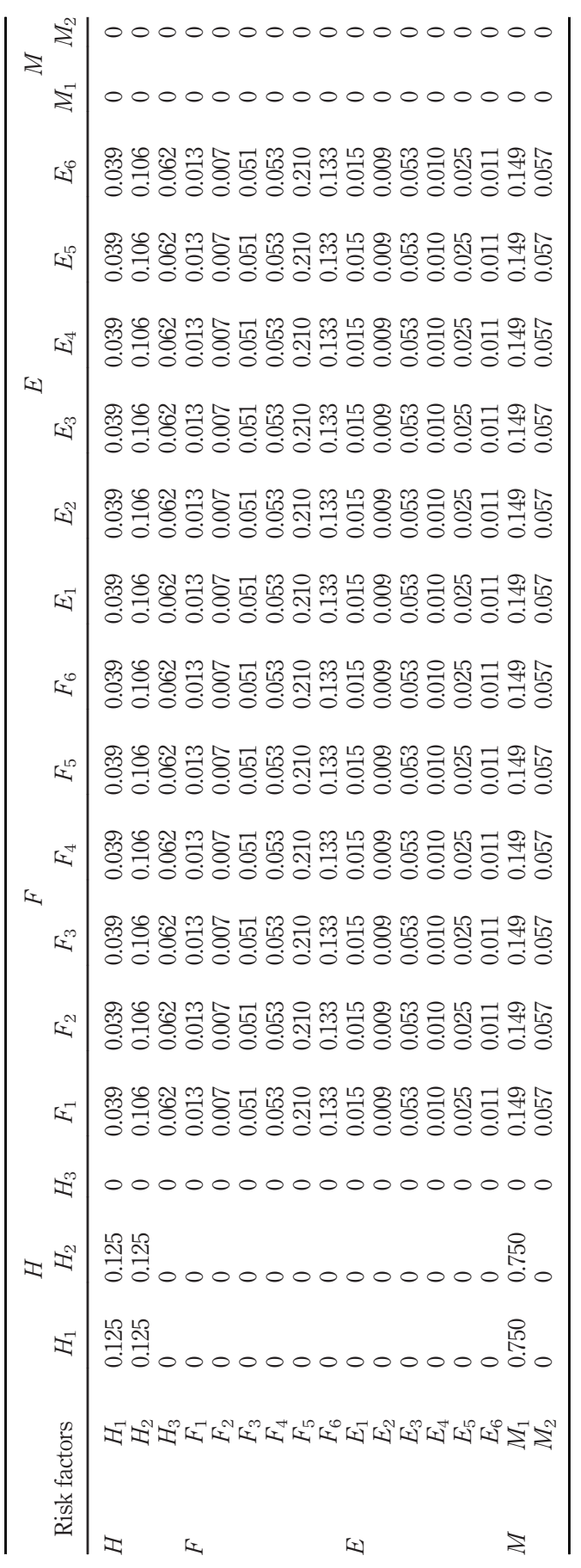

High-speed railway operation

Table 6.

Weighted over-limit super matrix of the secondary risk factors relative to the primary risk factors 
3,1

48

\begin{tabular}{lrrrr}
\hline & & & Expert comment & \\
Factor & $V_{1}$ & $V_{2}$ & $V_{3}$ & $V_{4}$ \\
\hline$H_{1}$ & 6 & 4 & 4 & 1 \\
$H_{2}$ & 9 & 3 & 1 \\
$H_{3}$ & 6 & 8 & 1 & 0 \\
$F_{1}$ & 3 & 8 & 4 & 0 \\
$F_{2}$ & 6 & 5 & 1 & 2 \\
$F_{3}$ & 5 & 3 & 5 & 0 \\
$F_{4}$ & 5 & 4 & 6 & 2 \\
$F_{5}$ & 6 & 4 & 3 & 0 \\
$F_{6}$ & 3 & 5 & 7 & 0 \\
$E_{1}$ & 9 & 5 & 1 & 0 \\
$E_{2}$ & 4 & 8 & 3 & 0 \\
$E_{3}$ & 4 & 4 & 6 & 0 \\
$E_{4}$ & 11 & 4 & 2 & 2 \\
$E_{5}$ & 8 & 5 & 0 & 2 \\
$E_{6}$ & 10 & 5 & 5 & 0 \\
$M_{1}$ & 3 & 5 & 5 & 0 \\
$M_{2}$ & 5 & 3 & & \\
\hline
\end{tabular}

Figure 4.

Weight distribution of primary evaluation factors

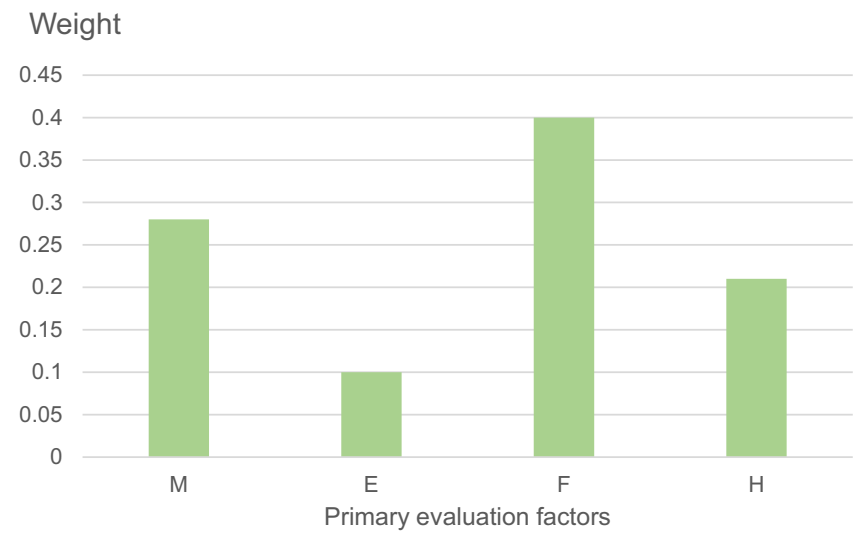

manner; the signal and control system of high-speed trains is mainly used for dispatching and commanding and automatic control of trains. At the same time, when the train encounters severe weather, sudden natural disasters or platform emergencies, the train control system can take corresponding measures to avoid accidents, so it is the core technical equipment to ensure safe driving and improve operational efficiency.

\subsection{Determine the evaluation matrix}

To illustrate the practical application of the fuzzy network analysis method, a calculation example is provided for reference. For the high-speed railway line $\mathrm{S}$, a total of 15 experts were invited to determine the degree of $\mathrm{S}$ subordination to the comment set $\mathrm{V}$ based on 17 secondary evaluation factors. A total of 15 questionnaires were issued, 15 questionnaires were returned, and 15 valid questionnaires were received. The recovery rate and effective rate were both $100 \%$. The reliability test coefficient of the questionnaire results Cronbach's 


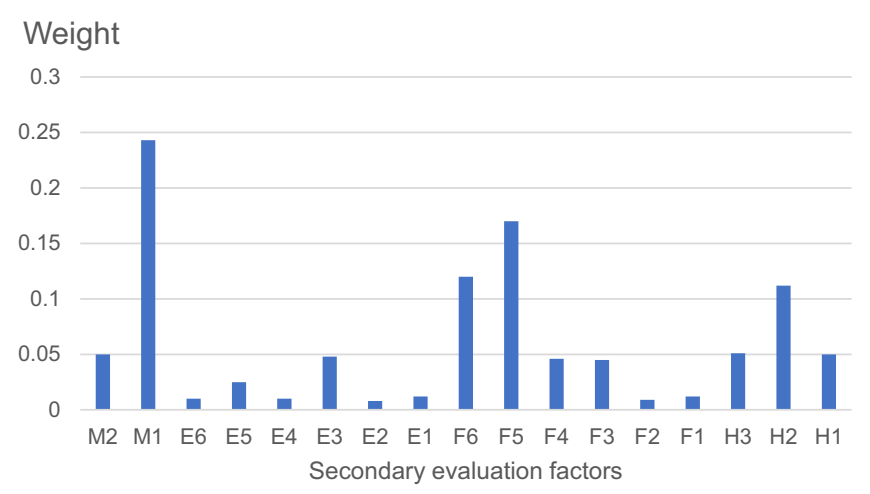

High-speed railway operation

Figure 5.

Weight distribution of secondary evaluation factors

$\alpha=0.809>0.800$, indicating that the questionnaire is internally consistent. The frequency distribution of expert questionnaire survey results is shown in Table 6.

According to the frequency distribution table of the statistical survey results, the evaluation matrix $R$ is obtained:

$H_{1}$
$H_{2}$
$H_{3}$
$F_{1}$
$F_{2}$
$F_{3}$
$F_{4}$
$F_{5}$
$F_{6}$
$E_{1}$$\quad\left[\begin{array}{ccccc}0.400 & 0.267 & 0.267 & 0.067 \\ 0.133 & 0.600 & 0.200 & 0.067 \\ 0.400 & 0.533 & 0.067 & 0 \\ 0.200 & 0.533 & 0.267 & 0 \\ E_{2} & 0.400 & 0.333 & 0.067 & 0.200 \\ E_{3} & 0.333 & 0.200 & 0.333 & 0.133 \\ E_{4} & 0.333 & 0.267 & 0.400 & 0 \\ E_{5} & 0.400 & 0.267 & 0.200 & 0.133 \\ E_{6} & 0.200 & 0.333 & 0.467 & 0 \\ M_{1} & 0.600 & 0.333 & 0.067 & 0 \\ M_{2} & 0.267 & 0.533 & 0.200 & 0 \\ 0.267 & 0.267 & 0.400 & 0.067 \\ 0.733 & 0.267 & 0 & 0 \\ 0.533 & 0.333 & 0.133 & 0 \\ 0.667 & 0.333 & 0 & 0 \\ 0.200 & 0.333 & 0.333 & 0.133 \\ 0.333 & 0.200 & 0.333 & 0.133\end{array}\right]$


SRT

3,1

\subsection{Fuzzy comprehensive evaluation}

Fuzzy transform the evaluation matrix $R$ and the weight vector $\mathrm{A}: \mathrm{B}=\mathrm{A} \times \mathrm{R}$, where the fuzzy operator $\times$ adopts the $\mathrm{M}(+, \bullet)$ operator (Lijuan and Qiao, 2012), that is, the weighted average method, and the operation rule of this operator is $b_{j}=\Sigma a_{i} \bullet r_{i j}$. Finally, the evaluation result $B=(0.294,0.342,0.282,0.082)$ is obtained. According to the principle of maximum membership degree, it can be known that the comprehensive safety evaluation result of high-speed railway line S is "safer".

\section{Conclusion}

Through literature investigation and field investigation, combined with high-speed railway safety key points and system composition, 4 first-level indicators and 17 second-level indicators were selected to construct a high-speed railway operation safety evaluation index system.

In view of the complex structure of the high-speed railway system and the high degree of coupling of fault factors, 17 secondary safety factors are analyzed through the ANP, and the relative importance of each evaluation factor to the operational safety of high-speed railways is obtained. It can be seen from the results that the personnel management system and the signal and control system have the largest weight.

On the basis of establishing an evaluation index system, comprehensively considering the internal coupling relationship between evaluation indexes and the fuzziness of highspeed railway operation safety evaluation, high-speed railway uses ANP fuzzy network analysis method to construct high-speed railway operation, and the safety evaluation model has certain advantages and practicability in the case of the relative lack of high-speed railway operation data and fault data.

\section{References}

Chen, S., Guohua, F. and Xianfeng, H. (2018), “Application of fuzzy network analytic hierarchy process in risk assessment of water conservancy project bidding", Water Conservancy Economics, Vol. 36 No. 2, pp. 15-19, 83.

Du, D., Qinghua, P., and Yan, W. (2015), Modern Comprehensive Evaluation Methods and Case Selection, 3rd ed., Tsinghua University Press, Beijing, pp. 37-62.

Feng, Y., Li, X., Li, X. and Jiang, A. (2013), "Interrelationship analysis of high-speed railway safety influence factors based on structural equation”, Journal of Beijing Jiaotong University (Social Science Edition), Vol. 12 No. 4, pp. 75-83, 108.

Guo, M., Wei, W., Liao, G. and Chu, F. (2016), "The impact of personality on driving safety among Chinese highspeed railway drivers", Accident Analysis and Prevention, Vol. 92 No. 7, pp. 9-14.

He, X., Qiang, W., Lei, X. and Chen, H. (2018), "Research on the effectiveness evaluation of pontoon bridge equipment system based on F-ANP", Ship Electronic Engineering, Vol. 38 No. 6, pp. 81-85.

Lijuan, X. and Qiao, C. (2012), "The selection of composition operator in fuzzy comprehensive evaluation”, Forum for Science and Technology Association (Second Half of the Month), No. 9, pp. 103-104.

Lisha, P., Long, C., Lan, L., Bo, C. and Yong, J. (2015), "Safety evaluation of urban rail transit stations based on analytic hierarchy process and entropy method", Urban Rail Transit Research, Vol. 18 No. 9, pp. 48-51.

Qian, W., Qi, T., Zhao, Y., Le, Y. and Yi, H. (2019), "Deformation characteristics and safety assessment of a high-speed railway induced by undercutting metro tunnel excavation", Journal of Rock Mechanics and Geotechnical Engineering, Vol. 11 No. 1, pp. 88-98.

Qin, C. (2018), Research on the Quality Evaluation of Middle-Level Managers of Power Supply Enterprises in Prefecture and City Based on F-ANP, North China Electric Power University, Beijing. 
Renchao, Z. and Dengbo, G. (2017), Introduction to High-Speed Railway, Southwest Jiaotong University Press, Chengdu.

Shi, L. and Jian, W. (2018), "Research on risk assessment of wireless block center based on network AHP and evidence theory", Urban Rail Transit Research, Vol. 21 No. 12, pp. 18-23.

Sishuai, Z. (2011), Evolution Mechanism of High-Speed Railway Operation Accident Based on Dissipative Structure, Beijing Jiaotong University, Beijing.

Wang, J. and Jianbin, M. (2019), "Risk assessment of bridge ship collision based on fuzzy network analysis", China Water Transport (the Second Half of the Month), Vol. 19 No. 2, pp. 86-88.

Wu, X., Wu, Y., Ma, W., Mao, T. and Lu, T. (2018), "Evaluation of marine emergency aid ability based on fuzzy network analysis", Journal of Wuhan University of Technology (Information and Management Engineering Edition), Vol. 40 No. 4, pp. 383-388, 395.

Xu, Z.W., Wu, S.C. and Wang, X.S. (2019), "Fatigue evaluation for high-speed railway axles with surface scratch", International Journal of Fatigue, Vol. 123 No. 6, pp. 79-86.

\section{Corresponding author}

Yichuan Pen can be contacted at: Yichuanpeng1982@hotmail.com

For instructions on how to order reprints of this article, please visit our website: 